\title{
The role of intraoperative ultrasound for the assessment of the focal liver lesions in patients with colorectal cancer
}

\author{
Claudiu-Dumitru Damian¹, Nicolae Rednic ${ }^{2}$, Doru Munteanu ${ }^{3}$, Mircea Cazacu ${ }^{4}$
}

${ }^{1} \mathrm{PhD}$ student, "Iuliu Hațieganu" University of Medicine and Pharmacy, Scandia Imagistica, Cluj-Napoca, Romania ${ }^{2} 4^{\text {th }}$ Internal Medicine Department, Railway University Hospital Cluj-Napoca, Romania

${ }^{3}$ Radiology and Imaging Department, Railway University Hospital Cluj-Napoca, Romania

${ }^{4} 4^{\text {th }}$ Surgical Department, "Iuliu Haţieganu" University of Medicine and Pharmacy, Cluj-Napoca, Romania

\begin{abstract}
Objective: To evaluate the concordance between intraoperative ultrasound (IOUS) and abdominal ultrasound (US) for the characterization of focal liver lesions in patients with colorectal cancer, and to evaluate the way in which IOUS influences the initially established hepatic surgical protocol based on abdominal US only. Method: In this prospective study 73 patients with colorectal cancer and focal liver lesions found during IOUS were included. Abdominal US and IOUS findings were compared. The initially established hepatic surgical protocol based on abdominal US only was recorded. All patients in whom IOUS influenced this protocol and the way in which this was influenced were recorded. Results: Of the 73 patients, 41 (56.2\%) had focal liver lesions classified as malignant by IOUS. In 26 of the 73 patients $(35.6 \%)$, there were discordances between abdominal US and IOUS. IOUS influenced the operative protocol in 9 of the 73 patients included in the study $(12.3 \%)$, and in 8 of the 41 patients with malignant lesions $(19.5 \%)$. IOUS influenced the operative protocol by cancelling the preoperative hepatic surgery decision by evidencing the additional multiple malignant focal liver lesions, following the classification of some lesions as benign or by demonstrating the non-resectable character of other lesions. IOUS also determined unplanned hepatic resections by detecting resectable malignant lesions undiagnosed by abdominal US, and extended or limited the scheduled hepatic surgery based on abdominal US only. Conclusions: There were inconsistencies between IOUS and abdominal US, therefore in patients with colorectal cancer IOUS provided additional information about focal liver lesions. IOUS influenced the operative protocol and consequently, unnecessary liver surgery was avoided. Also, IOUS identified malignant resectable liver lesions which were undetected by abdominal US.
\end{abstract}

Keywords: intraoperative ultrasound, colorectal cancer, focal liver lesions, hepatic surgery.

\section{Introduction}

Intraoperative ultrasound (IOUS) was initially used by Schlegel in 1961 for the detection of kidney stones, and subsequently, by Knight and Newell in 1963, for common bile duct stones [1]. Onik et al [2] showed that IOUS can guide hepatic criosurgery ablation - that was the beginning of using IOUS in liver oncologic surgery.

Received 01.02.2014 Accepted 9.04.2014

Med Ultrason

2014, Vol. 16, No 2, 114-118

Corresponding author: Claudiu-Dumitru Damian

4/51Aleea Padis str

400452 Cluj-Napoca, Romania

Phone: +40727803804

E-mail: dr_damian_claudiu@yahoo.com
The liver is known to be the first location for metastases of abdominal cancers [3]. Until the advent of multidetector CT and MRI, IOUS was considered to identify with $20-30 \%$ more liver lesions than preoperative imaging [4]. Today IOUS identifies undetected preoperative liver lesions measuring $1 \mathrm{~cm}$ or less in diameter $(5-15 \%$ more lesions than preoperative imaging) [5,6].

IOUS is irreplaceable in the characterization of small size liver lesions due to its excellent spatial resolution. Small cysts are easily detected by IOUS and are difficult to differentiate from malignant lesions by $\mathrm{CT}$, due to the partial volume effect. Small size hemangiomas are easily characterized by IOUS due to homogeneous hyperechogenicity, without a peripheral halo $[7,8]$. IOUS is considered to be the best method for evaluating the relation be- 
tween the liver lesions and the nearby vascular structures and biliary ducts [9]. Also, the IOUS has an impact on surgical decision in $32-51 \%$ of the patients proposed for hepatic surgery (for primary or secondary tumors) [1].

The first objective of this study was to evaluate the concordance between IOUS and abdominal ultrasound (US) for the characterization of focal liver lesions in patients with colorectal cancer. The second objective was to evaluate the way in which IOUS influenced the initially established hepatic surgical protocol based on abdominal US only.

\section{Material and method}

We performed a prospective study between October 2007-December 2010 enrolling 73 patients diagnosed with colorectal cancer undergoing surgery in the $4^{\text {th }}$ Surgical Department of "Iuliu Haţieganu" University of Medicine and Pharmacy, Cluj-Napoca, Romania. Written, informed consent for participation was obtained from each subject prior to enrolment. The Ethics Committee of the University approved the study protocol.

The inclusion criteria were patients diagnosed with colorectal cancer (colonoscopy with biopsy) scheduled for surgery treatment and focal liver lesions found during IOUS. Biological tests were carried out for all patients. All the patients were preoperative evaluated by abdominal ultrasound (US). From the patients with colorectal cancer undergoing surgery in the aforementioned period (247 patients) in 73 patients focal liver lesions were detected at IOUS and the patients were included in study.

During IOUS (Aloka SSD4000, linear transducer with variable frequency $5-10 \mathrm{MHz}$ ), the liver was scanned from the left (at the level of the liver dome) to the right margin, with the transducer in transverse plane, following a cranial to caudal direction, with the superposition of each scanning plane over the previous one. The hepatic IOUS characterized each detected focal liver lesion, with malignancy/benignity classification, recording the segmental liver location and maximal size. The focal lesions with transonic content (cysts) (fig 1, fig 2) and homogeneous hyperechogenic nodular lesions without a peripheral halo (hemangiomas) were classified as benign. The hypoechogenic nodular lesions and lesions with a peripheral halo were classified as malignant (fig 3, fig 4). The lesions with a diffuse arrangement (steatosis changes) were not included in this study.

The results of IOUS were compared to that of abdominal US preoperatively findings. Abdominal US characterized each detected focal liver lesion, with malignancy/ benignity classification (according to the criteria men-

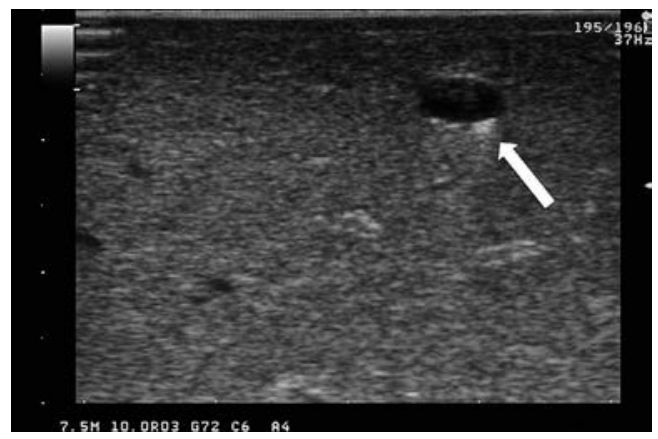

Fig 1. Hepatic cyst (arrow) detected by IOUS

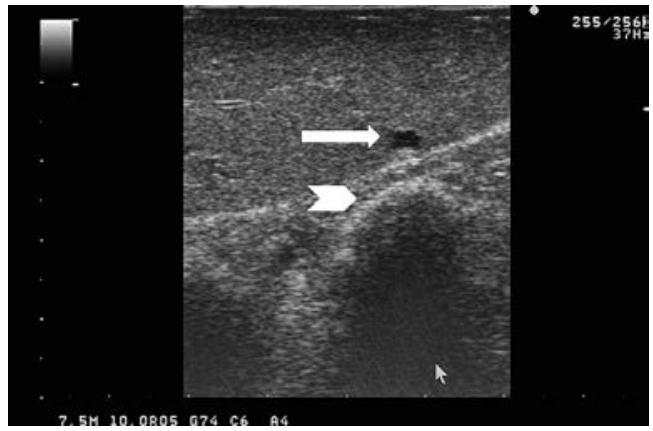

Fig 2. Palpable nodular hepatic lesion (surgeon finger - arrowhead) - diagnosed as cyst (arrow) by IOUS

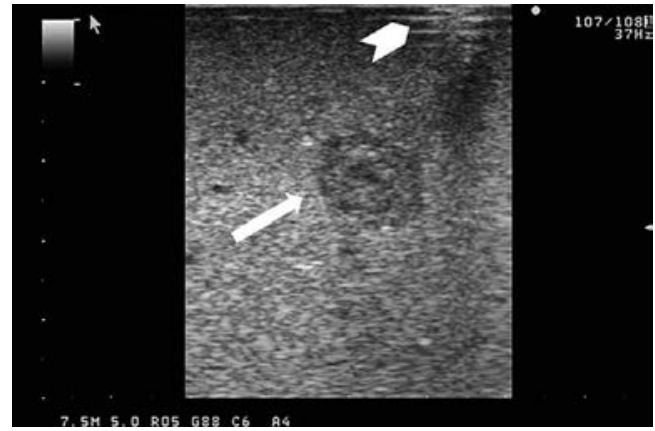

Fig 3. Hepatic metastasis (arrow) nearby an electrocautery mark (arrowhead)

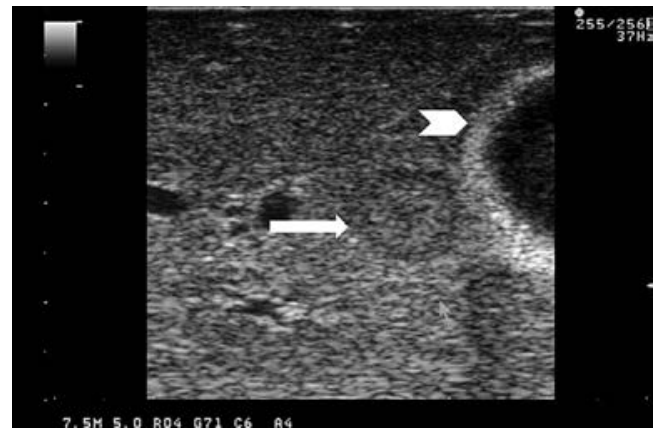

Fig 4. Hepatic metastasis (arrow) adjacent to the gallbladder (arrowhead) 
tioned for IOUS); the segmental liver location and maximal size were recorded.

According to the abdominal US, the option for liver resection and the extension of the considered resection was recorded preoperatively in all patients. All patients in whom IOUS influenced the preoperatively established hepatic surgical strategy and the way in which this was influenced were recorded.

\section{Statistical analysis}

The statistical analysis was performed using the SPSS 17.0 for Windows and Medcalc 10.3.0.0. For qualitative variables, the frequency, prevalence and cumulative percentage were calculated, and for quantitative variables, the mean and standard deviation, the median and confidence intervals were calculated. For the analysis of the differences between qualitative variables, the $\chi 2$ test was used. The normality of the distribution of continuous numerical variables was verified using the KolmogorovSmirnov and D'Agostino-Pearson tests. The differences between the means of continuous quantitative variables were evaluated using the (unpaired and paired) Student test, and for variables with distribution that do not respect the normality condition, non-parametric tests were used (Mann-Whitney U). The Kappa concordance coefficient was calculated: $p<0.05$ was considered statistically significant.

\section{Results}

The study group was formed by 35 females (age range $34-80$ years) and 38 males (age range $40-82$ years).

Of the 73 patients included in the study, in 41 patients the focal liver lesions found at IOUS (single lesion in 9 patients, two focal lesions in 3 patients, and multiple focal lesions in 29 patients) were classified as malignant. Three of these patients (7.3\%) presented associated benign focal liver lesions. The focal liver lesions found in 32 patients were considered benign by IOUS.

Comparing the results of IOUS with preoperative US and using the concordance test, the Kw index had a value of 0.64 for the concordance between abdominal US and IOUS.

The results of IOUS and abdominal US in characterization the liver focal lesions are summarized in Table I.

There were discordances between the number of focal lesions identified by IOUS and abdominal US in 7 patients with malignant liver lesions and in 2 patients with benign liver lesions (not taking into account the 5 patients with malignant lesions and 11 patients with benign lesions failed to visualized by abdominal US).

The information provided by IOUS influenced the protocol of hepatic surgery according to abdominal US imaging in 9 of the 73 patients included in the study $(12.3 \%)$ (table II), respectively in 8 of the 41 patients with malignant liver lesions at IOUS (19.5\%). Of $12 \mathrm{pa}-$ tients with one or two malignant hepatic lesions IOUS influenced the operative protocol in 6 patients $(50 \%)$. In 4 patients IOUS cancelled the hepatic surgery decision based on the abdominal US diagnosis. Of the 4 patients, in 1 patient IOUS refuted the abdominal US diagnosis of malignant focal liver lesion, establishing benignity diagnoses; in 1 patient, IOUS demonstrated the inoperability of the malignant liver lesion due to the vascular extension that was preoperatively understaged; in 2 patients, IOUS demonstrated the presence of multiple malignant liver lesions.

Of the 41 patients with malignant liver lesions, 8 patients underwent resection of the focal liver lesions. In 3 of the 8 patients, the surgical procedure was made according to the abdominal US findings, the surgery being facilitated by IOUS. In these cases, IOUS set the relation between the liver lesions and the nearby vascular structures. In 3 of the 8 patients, the hepatic surgery decision was made during the intervention for colorectal cancer, following the IOUS demonstration of focal liver lesions undetected by abdominal US. In 1 patient, surgery was limited compared to the preoperatively established protocol - abdominal US detected two malignant liver lesions, while IOUS detected only one, which was resected. In 1 patient, hepatic surgery was extended compared to the

Table I. The results of IOUS and abdominal US in characterization the liver focal lesions.

\begin{tabular}{ll}
\hline IOUS & Abdominal US \\
\hline $\mathbf{4 1}$ patients with malignant lesions & $\mathbf{3 6}$ patients with malignant lesions \\
& $\mathbf{5}$ patients with no liver lesions \\
\hline $\mathbf{3 2}$ patients with benign lesions & $\mathbf{1}$ patient with malignant lesions \\
& $\mathbf{2 0}$ patients with benign lesions \\
& $\mathbf{1 1}$ patients with no liver lesions \\
\hline
\end{tabular}


Table II. Cases influenced by IOUS findings concerning hepatic surgery protocol

\begin{tabular}{|c|c|c|}
\hline Hepatic surgery protocol according to abdominal US findings & Hepatic surgery protocol modified after IOUS & Patients \\
\hline \multirow[t]{2}{*}{ Without hepatic intervention } & resection & 3 \\
\hline & extended & 1 \\
\hline \multirow[t]{2}{*}{ Hepatic resection } & limited & 1 \\
\hline & cancelled & 4 \\
\hline total & & 9 \\
\hline
\end{tabular}

preoperatively established protocol - abdominal US detected one malignant liver lesion, IOUS detected one additional malignant lesion, and the resection of both liver lesions was carried out.

\section{Discussion}

IOUS is considered by the literature to be the imaging exploration with the highest resolution in the examination of the liver. It allows for a very good characterization of focal liver lesions, with both sensitivity and specificity values over $90 \%$ [10-14]. Of the 73 patients included in the study in $41(56.2 \%)$ patients the focal liver lesions found at IOUS were classified as malignant and in 32 $(43.8 \%)$ patients the focal liver lesions found at IOUS were classified as benign. The high percentage of benign lesions was attributable to the patient inclusion criterion: the presence of any focal liver lesion on IOUS; this approach started from the premise of a lower specificity of preoperative imaging techniques. Literature studies frequently exclude patients with focal liver lesions considered benign by preoperative imaging and only include patients with a suspicion of malignant lesions - the frequency of benign liver lesions described by the literature is $10-30 \%[10,15]$. The increase in the number of benign lesions in the study group is correlated with the reduction of the specificity of preoperative imaging [15]. In the literature, discordances between IOUS and preoperative imaging range between $23-32 \%[16,17]$. Our study found discordances between IOUS and abdominal US in 26 of the 73 patients $(35.6 \%)$, while the values of the $\mathrm{Kw}$ concordance index were 0.64 (the values of the $\mathrm{Kw}$ concordance index are considered to be good within the [0.6-0.8] interval and very good within the [0.8-1.0] interval). The discordance index found in our study may be due to the great number of patients with benign liver lesions but this value indicates the need for IOUS for the additional characterization of focal liver lesions in patients with colorectal cancer.

In our study, IOUS influenced the operative protocol established according to abdominal US imaging in
9 patients $(12.3 \%$ of the patients included in the study). In the literature, IOUS changes the operative protocol in variable proportions, ranging within the $10-86 \%$ interval [10,18-20], depending on patient selection and the reference standard - patients with colorectal cancer or patients operated for secondary hepatic colorectal tumors. In our study, the $12.3 \%$ value was obtained by relating the number of patients in whom IOUS influenced the hepatic operative protocol to the total number of patients. Taking into consideration only the 41 patients with malignant lesions detected by IOUS in whom the operative protocol was influenced, a $19.5 \%$ value was obtained; in $50 \%$ of patients with a single or double malignant hepatic lesion (which was potentially resectable) IOUS influenced the operative protocol, which emphasizes once more the importance of IOUS for establishing intraoperative surgical strategy in these patients. In our study, IOUS influenced the operative protocol by cancelling the preoperative hepatic surgery decision by evidencing the additional multiple malignant focal liver lesions, following the classification of some lesions as benign or by demonstrating the non-resectable character of other lesions. IOUS also determined unplanned hepatic resections by detecting resectable malignant lesions undiagnosed by abdominal US, and extended or limited the scheduled hepatic surgery.

Among the limitations of the study, we should mention in the first place the absence of histopathological diagnosis for all hepatic lesions; such a study can only be carried out in the case of patients with liver transplantation, in order to subsequently perform a complete examination of the entire liver parenchyma. Other limitations of the study is the lack of contrast enhanced ultrasonography examinations prior and during surgery and the absence of a comparative study between IOUS and preoperative contrast enhanced computed tomography (CECT). There are published studies $[18,21]$ that showed the role of contrast enhanced intraoperative ultrasound in the identification of supplementary focal liver lesions and in the differentiation of nodular liver lesions. Comparative study of IOUS and CECT will be the subject of another study. 


\section{Conclusions}

There were inconsistencies between IOUS and abdominal US; therefore, in patients with colorectal cancer, IOUS provided additional information about focal liver lesions. IOUS influenced the operative protocol and consequently, unnecessary liver surgery was avoided. Also, IOUS identified malignant resectable liver lesions which were undetected by abdominal US.

\section{Conflict of interest: none}

\section{References}

1. Luck AJ, Maddern GJ. Intraoperative abdominal ultrasonography. Br J Surg 1999; 86: 5-16.

2. Onik G, Kane R, Steele G, et al. Monitoring hepatic cryosurgery with sonography. AJR Am J Roentgenol 1986; 147: 665-669.

3. DeOliveira ML, Pawlik TM, Gleisner AL, Assumpcaom L, Lopes-Filho GJ, Choti MA. Echogenic appearance of colorectal liver metastases on intraoperative ultrasonography is associated with survival after hepatic resection. J Gastrointest Surg 2007; 11: 970-976.

4. Soyer P, Mosnier H, Choti MA, Rymer R. Intraoperative and laparoscopic sonography of the liver. Eur Radiol 1997; 7: 1296-1302.

5. Kane RA. Intraoperative ultrasound. In: Bluth EI, Benson CB, Ralls PW, Siegel MJ, eds. Ultrasonography in urology. Second edition. New York: Thieme, 2008: 123-139.

6. Ellsmere J, Kane R, Grinbaum R, Edwards M, Schneider $\mathrm{B}$, Jones D. Intraoperative ultrasonography during planned liver resections: why are we still performing it? Surg Endosc 2007; 21: 1280-1283.

7. Lee RA, Kane RA, Charboneau JW. Intraopeartive and laparoscopic sonography of the abdomen. In: Rumack CM, Wilson SR, Charboneau JW, Johnson JAM, eds. Diagnostic ultrasound, vol I. Third edition. St. Louis: Elsevier-Mosby, 2005: 705-734.

8. Badea RI, Dudea SM, Mircea PA, Stamatian F, eds. Tratat de ultrasonografie clinică, vol I. Bucureşti: Editura Medicală, 2004: 724.

9. Appelbaum L, Kruskal JB, Sosna J, Kane RA. Intraoperative Ultrasonography of the Liver: Why, When, and How? Ultrasound Clin 2006; 1: 511-520.
10. Zacherl J, Scheuba C, Imhof M, et al. Current value of intraoperative sonography during surgery for hepatic neoplasms. World J Surg 2002; 26: 550-554.

11. Bhattacharjya S, Bhattacharjya T, Baber S, Tibballs JM, Watkinson AF, Davidson BR. Prospective study of contrast-enhanced computed tomography, computed tomography during arterioportography, and magnetic resonance imaging for staging colorectal liver metastases for liver resection. Br J Surg 2004; 91: 1361-1369.

12. Machi J, Sigel B, Zaren HA, Kurohiji T, Yamashita Y. Operative ultrasonography during hepatobiliary and pancreatic surgery. World J Surg 1993; 17: 640-645.

13. Guimarães CM, Correia MM, Baldisserotto M, de Queiroz Aires EP, Coelho JF. Intraoperative ultrasonography of the liver in patients with abdominal tumors: a new approach. $\mathrm{J}$ Ultrasound Med 2004; 23: 1549-1555.

14. Kruskal JB, Kane RA. Intraoperative US of the liver: techniques and clinical applications. Radiographics 2006; 26 : 1067-1084.

15. Meijer S, Paul MA, Cuesta MA, Blomjous J. Intra-operative ultrasound in detection of liver metastases. Eur J Cancer 1995; 31A: 1210-1211.

16. D'Onofrio M, Gallotti A, Martone E, et al. Is intraoperative ultrasound (IOUS) still useful for the detection of liver metastases? J Ultrasound 2009; 12: 144-147.

17. Sietses C, Meijerink MR, Meijer S, van den Tol MP. The impact of intraoperative ultrasonography on the surgical treatment of patients with colorectal liver metastases. Surg Endosc 2010; 24: 1917-1922.

18. Torzilli G, Botea F, Procopio F, et al. Use of contrast-enhanced intraoperative ultrasonography during liver surgery for colorectal cancer liver metastases - Its impact on operative outcome. Analysis of a prospective cohort study. EJC Supplements 2008; 6: 16-23.

19. Kulig J, Popiela T, Kłek S, et al. Intraoperative ultrasonography in detecting and assessment of colorectal liver metastases. Scand J Surg 2007; 96: 51-55.

20. Piccolboni D, Ciccone F, Settembre A, Corcione F. Liver resection with intraoperative and laparoscopic ultrasound: report of 32 cases : Ultrasonic shears device for liver parenchymal transection. Surg Endosc 2008; 22: 1421-1426.

21. Hohmann J, Skrok J, Basilico R, et al. Characterisation of focal liver lesions with unenhanced and contrast enhanced low MI real time ultrasound: on-site unblinded versus off-site blinded reading. Eur J Radiol 2012; 81: e317-e24. 